\section{Reply to: Saphenous sparing laser modern options (com- ment to Gianesini et al., Veins and Lymphatics 2013;2:e21 and Veins and Lymphatics 2015;4:5246)}

\section{Sergio Gianesini}

Vascular Disease Center, University of Ferrara, Cona (FE), Italy

Dear Editor,

I really enjoyed Dr. Ferracani interest in saphenous sapring innovations. ${ }^{1}$

As a matter of fact modern Phlebology is constantly offering us brand new tools to improve our outcomes. Nevertheless, these better outcomes regard just a better mini-invasiveness, that is not followed by a corresponding improvement in the post-procedural recurrence rate. As pointed out by the recent PREVAIT analysis on the recurrence rate in fact traditional stripping, radiofrequency and laser procedure produce overlapping outcomes in terms of recurrences. ${ }^{2}$

This feature is testified by the modern guidelines recommending endovenous thermal ablation over surgery not because of a better recurrence rate performance, but because of their mini-invasiveness. ${ }^{3}$

At the same time, this year a Cochrane review reported a varicose veins recurrence reduction following a saphenous sparing surgical strategy rather than a traditional ablative one. ${ }^{4}$

Whenever dealing with saphenous sparing options a great attention must be paid. It has been demonstrated that a hemodynamic procedure performed in not well trained centres leads to outcomes that are worse than a traditional ablative procedure. ${ }^{5}$

For this reason I hope Dr. Ferracani will soon produce a paper with a clear indication of the hemodynamic types that are eligible for his procedure, specifying the required learning curve for such an elegant and interesting therapeutic option.

Once again a clear distinction must be made among the strategy and the technique.

Along these last decades Phlebology has moved its therapeutic army endowing it with always brand new technical devices. Nevertheless the strategy has always remained ablative.

Previous surgical papers demonstrated the competitiveness of a saphenous sparing therapeutic, so forcing our Discipline to evaluate the feasibility and performance of the new devices not only as ablative tools. In conclusion, looking for a recurrence rate reduction, it's desirable to explore the performance not just of new tools in an old strategy, rather innovative tools for innovative strategies.

Only in this way I think we'll be able to appreciate the new devices at their best, finally improving not just our mini-invasiveness, but also the recurrence rate.

\section{References}

1. Ferracani EL. Saphenous sparing laser modern options (comment to Gianesini et al., Veins and Lymphatics 2013;2:e21 and Veins and Lymphatics 2015;4:5246). Veins and Lymphatics 2015;4:5531.
Correspondence: Sergio Gianesini, Vascular Disease Center, University of Ferrara, via Aldo Moro 8, 44128 Cona (FE), Italy.

E-mail: sergiogianesini@hotmail.com

Key words: Letter to editor; saphenous sparing procedures.

Received for publication: 30 September 2015. Accepted for publication: 30 September 2015.

This work is licensed under a Creative Commons Attribution 3.0 License (by-nc 3.0).

(C) Copyright S. Gianesini, 2015

Licensee PAGEPress, Italy

Veins and Lymphatics 2015; 4:5559

doi:10.4081/vl.2015.555.

2. Perrin M. Presence of varices after operative treatment: a review. Phlebolymphology 2014;21:1.

3. Gloviczki P, Comerota AJ, Dalsing MC, et al. The care of patients with varicose veins and associated chronic venous diseases: clinical practice guidelines of the Society for Vascular Surgery and the American Venous Forum. J Vasc Surg 2011;53:2S$48 \mathrm{~S}$.

4. Bellmunt-Montoya S, Escribano JM, Dilme J, Martinez-Zapata MJ. CHIVA method for the treatment of chronic venous insufficiency. Cochrane Database Syst Rev 2015;6:CD009648.

5. Milone M, Salvatore G, Maietta P, et al. Recurrent varicose veins of the lower limbs after surgery. Role of surgical technique (stripping vs. CHIVA) and surgeon's experience. G Chir 2011;32:460-3. 